\title{
Reverse Electrical Remodeling Assessed by High-Frequency QRS Dyssynchrony and QRS Duration
}

\author{
Pavel Leinveber ${ }^{1}$, Josef Halamek ${ }^{3}$, Pavel Jurak ${ }^{3}$, Magdalena Matejkova ${ }^{2}$, \\ Jolana Lipoldova ${ }^{1}$, Miroslav Novak ${ }^{1}$ \\ ${ }^{1}$ ICRC, Department of Cardio-angiology, St Anne's University Hospital, Brno, Czech Republic \\ ${ }^{2}$ ICRC, St Anne's University Hospital, Brno, Czech Republic \\ ${ }^{3}$ Institute of Scientific Instruments, CAS, Czech Republic
}

\begin{abstract}
Reverse electrical remodeling (RER) is usually assessed by measuring the QRS duration (QRSd) before and after cardiac resynchronization therapy (CRT) during the stimulator off. The ventricular electrical depolarization dyssynchrony (DYS) can be assessed directly from ECG by high-frequency $Q R S$ analysis. The purpose of the study was to compare the RER assessment by the DYS and QRSd changes in CRT patients.

Echocardiography and 12-lead ECG were conducted in 26 CRT recipients. The averaged VI-V6 QRS envelopes were calculated through frequency ranges from 150 to $950 \mathrm{~Hz}$. The DYS was assessed from the envelopes, and the QRSd was measured. All the ECG parameters were assessed before, after, and 6 months after the CRT during CRT off. Patients were divided to CRT responders/nonresponders by the change of end-systolic volume of the left ventricle in a 6-month follow-up.

Mean values of the DYS and QRSd parameters evince significantly shorter values in non-responders before CRT. When compared changes of DYS and QRSd, only DYS parameter is significantly shorter after 6-month follow-up compared with baseline DYS prior CRT.

The DYS parameter change as a marker of RER corresponds with reverse structural remodeling assessed by echocardiography.
\end{abstract}

\section{Introduction}

Cardiac remodeling refers to a structural modification of myocardium clinically manifesting as changes in size, structure, geometry, and function of the heart resulting from cardiac injury [1]. Pathological process of remodeling may be slowed down or even reversed by modern pharmacotherapy and/or by non-pharmacological methods. Cardiac resynchronization therapy (CRT) has been showed to induce reverse structural remodeling (RSR) in heart failure patients (HF) [2]. Since the CRT delivers its therapy through electrical bi-ventricular pacing, the possibility of the reverse electrical remodeling (RER), as a function of the QRS duration (QRSd) change, has also been previously investigated. Unfortunately, the results remain rather inconclusive. Several studies have demonstrated the RER of the native conduction with CRT [3, 4], but others have reported no positive shortening of the QRSd after the CRT $[5,6]$.

We have recently presented a novel method of an assessment of the ventricular electrical depolarization pattern [7]. In short, the ventricular electrical activation can be determined directly from the surface ECG V leads by the processing of the higher frequency components present in QRS complex. Their temporal distribution properties carry information about the timeline of an electrical activation and allow for the precise assessment of the possibly dyssynchronous depolarization of ventricles (DYS) in units of milliseconds.

The specific properties of the DYS parameter may also allow assessing the RER in CRT patients. The purpose of the study was to compare the RER assessment by the DYS and QRSd changes in CRT patients.

\section{Methods}

26 left bundle branch block (LBBB) consecutive CRT recipients (4 females, 19 dilated cardiomyopathies (DCM), 5 coronary artery diseases (CAD) and 2 coincidences of $\mathrm{DCM}+\mathrm{CAD})$ were included in the study. Echocardiography (Vivid E9, GE Healthcare, Wauwatosa, WI) and 5 min measurement of 12-lead ECG (SciSDA14, M\&I s r.o., Prague, Czech Republic) in calm supine position with sampling frequency $5 \mathrm{kHz}$ and resolution 24bits were conducted for further analysis.

The V1-V6 QRS depolarization envelopes maps were 
calculated for the passband width $100 \mathrm{~Hz}$ from 150 to $950 \mathrm{~Hz}$ with $100 \mathrm{~Hz}$ step using signal averaging technique to improve the signal-to-noise ratio as described in [7]. The DYS parameter was assessed automatically as an average of differences between soonest and latest activation in $\mathrm{V}$ leads' envelopes over the passbands. The activation difference was determined as an average of three methods: (1) time difference between 2 most distant maximums of the amplitude envelopes, (2) time difference between 2 most distant centers of gravity of the envelopes with $50 \%$ and (3) 30\% envelope base cut off (Figure 1-3. bottom black, green dashed, and green curve, respectively).

The standard QRSd was measured fully automatically using the custom-made software [8].

All the QRSd and DYS values were obtained before, 13 days after, and 6 months after the CRT in native conduction (during CRT off). Patients with a relative decrease of $10 \%$ and more in end-systolic volume (ESV) of the left ventricle after 6-month follow-up were considered CRT responders.

\section{Results}

Of all 26 patients were $18 \mathrm{CRT}$ responders by the improved ESV after 6 months of follow-up.

Mean values (Table 1.) of DYS and QRSd parameters were significantly lower in non-responders before CRT. DYS was also significantly lower in non-responders just after CRT, but QRSd was closely non-significant $(\mathrm{P}=0.06)$. Both DYS and QRSd were non-significant when measured during 6-month follow-up.

Tab. 1: Mean values of the DYS and QRSd parameters assessed before CRT implantation, just after the CRT implantation with CRT Off, and during 6-month follow-up with CRT Off divided into CRT responders (R) and nonresponders (NR). The values are represented as a mean \pm SD.

\begin{tabular}{llrrc}
\hline \multicolumn{2}{l}{ Mean values [ms] } & \multicolumn{1}{c}{ R } & \multicolumn{1}{c}{ NR } & p \\
\hline \multirow{2}{*}{ Before CRT } & DYS & $79 \pm 27$ & $52 \pm 20$ & $<0.05$ \\
& QRSd & $169 \pm 14$ & $155 \pm 14$ & $<0.05$ \\
After CRT Off & DYS & $77 \pm 26$ & $48 \pm 23$ & $<0.05$ \\
& QRSd & $168 \pm 16$ & $156 \pm 12$ & NS \\
6M CRT Off & DYS & $67 \pm 28$ & $51 \pm 19$ & NS \\
& QRSd & $165 \pm 20$ & $157 \pm 10$ & NS \\
\hline
\end{tabular}

There was a significantly lower DYS after 6-month follow-up compared to baseline DYS, while there was no DYS parameter change before and just after the CRT (Table 2.). No significant difference was found in QRSd just after CRT and 6-month follow-up against the baseline prior CRT (Table 3.). The data from 8 CRT non-responders did not show any significant differences of DYS or QRSd just after the CRT and 6-month follow-up compared to baseline values (Table 2 and 3.).

Tab. 2: Changes of the DYS parameter between three stages of the measurement - before CRT implantation, just after the CRT implantation with CRT Off, and during 6month follow-up with CRT Off divided into CRT responders $(\mathrm{R})$ and non-responders $(\mathrm{NR})$. The values are represented as a mean $\pm \mathrm{SD}$.

\begin{tabular}{lcc}
\hline DYS changes [ms] & R & NR \\
\hline \multirow{2}{*}{ After CRT Off - before CRT } & $-3 \pm 9$ & $-3 \pm 8$ \\
& NS & NS \\
6M CRT Off - before CRT & $-12 \pm 18$ & $-4 \pm 12$ \\
& $<\mathbf{0 . 0 1}$ & NS \\
6M CRT Off - after CRT Off & $-6 \pm 11$ & $0 \pm 12$ \\
& NS & NS \\
\hline
\end{tabular}

Tab. 3: Changes of the QRSd parameter between three stages of the measurement - before CRT implantation, just after the CRT implantation with CRT Off, and during 6month follow-up with CRT Off divided into CRT responders (R) and non-responders (NR). The values are represented as a mean $\pm \mathrm{SD}$.

\begin{tabular}{lcc}
\hline QRSd changes [ms] & R & NR \\
\hline \multirow{2}{*}{ After CRT Off - before CRT } & $0 \pm 4$ & $1 \pm 3$ \\
& $\mathrm{NS}$ & $\mathrm{NS}$ \\
6M CRT Off - before CRT & $-4 \pm 16$ & $2 \pm 8$ \\
& $\mathrm{NS}$ & $\mathrm{NS}$ \\
\multirow{2}{*}{ 6M CRT Off - after CRT Off } & $0 \pm 9$ & $1 \pm 7$ \\
& $\mathrm{NS}$ & $\mathrm{NS}$ \\
\hline
\end{tabular}
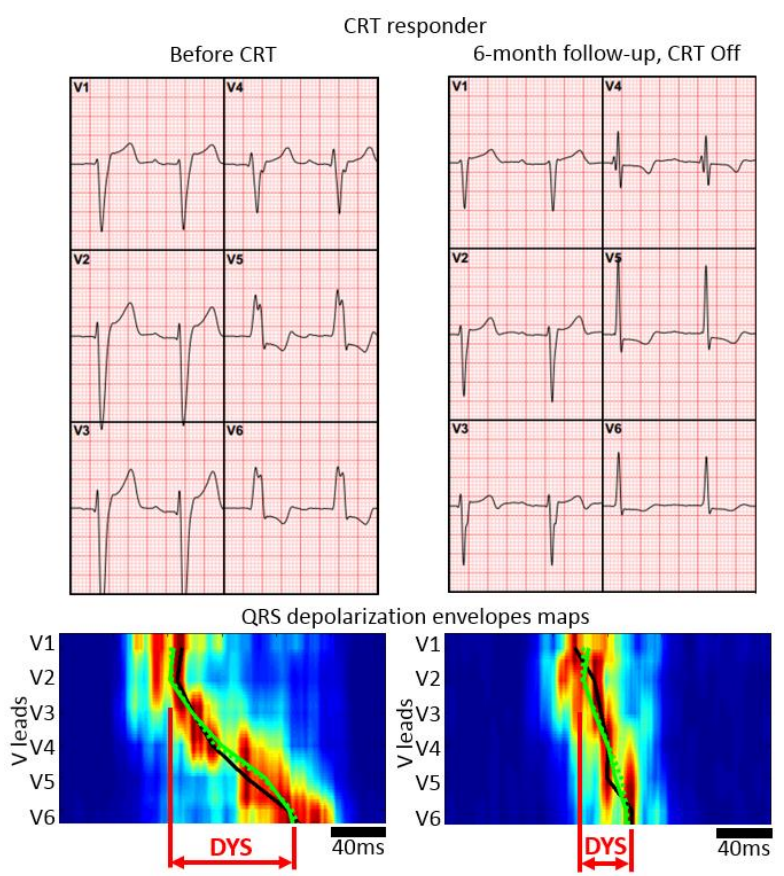

Figure 1. Example of the CRT responder by echocardiography (ESV increase by $12 \%$ ); with extensive 
shortening of QRSd from $177 \mathrm{~ms}$ to $121 \mathrm{~ms}$ and DYS from $112 \mathrm{~ms}$ to $43 \mathrm{~ms}$. QRS depolarization envelopes maps are created by amplitude normalization over the $\mathrm{V}$ lead envelopes (max. amplitude - red, min. amplitude - blue color), arranging envelopes into rows from V1 to V6 and performing a linear interpolation between the rows.

\section{Discussion}

Significant differences in the mean values of the DYS and QRSd parameters exist between CRT responders and non-responders defined by echocardiography. These findings are consistent with previously reported findings. Wider QRS is a known factor for positive CRT response [9]. We have recently shown, on part of the MADIT-CRT trial data, that higher baseline DYS values predict better benefit from CRT in LBBB patients [10]. The differences are also evident in the DYS and QRSd measured after biventricular pacemaker implantation during CRT Off, but only DYS varies significantly between responders and non-responders. QRSd differences slipped just below the significance $(p=0.06)$. The similar differences between responders and non-responders persist even after 6 months of CRT (during CRT Off) but are not significant enough.

The evaluation of the reverse structural remodeling after CRT is clinically well established [1] with an ongoing effort for a methodological improvement [11]. Unlike, the mechanism standing behind the reverse electrical remodeling as well as its diagnostic potential still remain rather unclear. Although the RER is primarily assessed by the change of the QRSd parameter, the non-existent unified methodology for the RER assessment could be one of the reasons.

Studies that assess the RER by comparing the QRSd during the biventricular pacing [12-14] report significant changes in QRSd before and after the CRT. But the majority of the published articles, evaluating RER, deal with the measurement of QRSd during native conduction before and after the CRT (CRT Off). Unfortunately, the reported results with native conduction vary considerably; from shortening the QRSd during follow-up by $11 \mathrm{~ms}$ [3] or $19 \mathrm{~ms}$ [4] to prolongation of the QRSd by $1 \mathrm{~ms}$ [5] or $6 \mathrm{~ms}$ [6]. Our results also did not find any significant differences in QRSd measured before CRT and during native conduction 6 months after CRT. The reason for such inconsistency between studies is unknown. It could be partially caused by using different methods of QRSd measurement. But there still remain other fundamental problems to be solved, such as whether to measure RER during biventricular pacing On or Off and the exact time course of measurement conduction (the long-term followup as well as the waiting period before measurement after the pacemaker settings change).

Ambiguous results of the RER assessment by intrinsic
QRSd open a question whether the QRSd parameter is sensitive and specific enough to securely evaluate RER. Our recently introduced DYS parameter [7] showed the significant reduction of the native dyssynchronous depolarization after 6 months of CRT despite no change in QRSd (An example in Figure 2.). The QRSd parameter represents the whole activation of the ventricles and it does not necessarily reflect dyssynchronous activation via bundle branch blockades (BBB). On the other hand, the DYS parameter, by its nature, is able not only clearly distinguish BBB but also evaluate any activation changes in $\mathrm{BBB}$ if present after the follow-up.
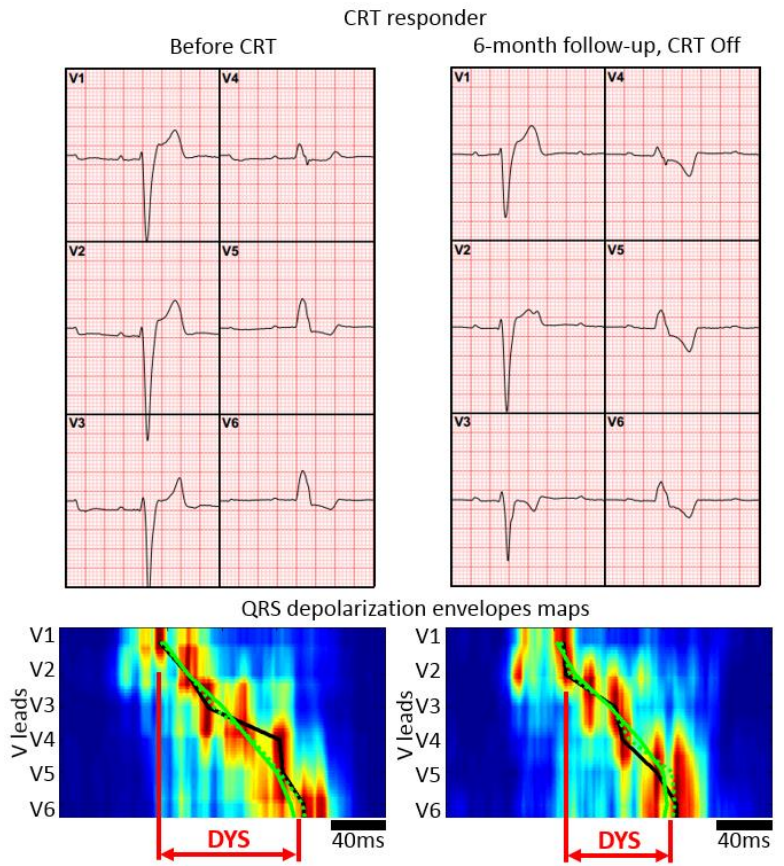

Figure 2. Example of the CRT responder by echocardiography (ESV increase by $27 \%$ ); with almost no change of QRSd (193ms to $191 \mathrm{~ms})$, but the substantial decrease of DYS (109ms to $79 \mathrm{~ms}$ ). QRS depolarization envelopes maps are created by amplitude normalization over the $\mathrm{V}$ lead envelopes (max. amplitude - red, min. amplitude - blue color), arranging envelopes into rows from V1 to V6 and performing a linear interpolation between the rows.

The DYS changes have not been found in CRT nonresponders (An example in Figure 3.), except one $\mathrm{DCM}+\mathrm{CAD}$ patient (ESV increase by only $5 \%$ ), whose DYS shortened from $48 \mathrm{~ms}$ to $22 \mathrm{~ms}$ with simultaneous shortening of the QRSd only by $7 \mathrm{~ms}$ (166 to $159 \mathrm{~ms}$ ) after the follow-up. Partial disagreement between RSR and RER has also been presented in [4].

\section{Conclusion}


The changes of the DYS parameter show a significant shortage of the ventricular conduction delay in CRT responders during native conduction, whereas the QRSd does not evince any significant changes. The DYS change as a marker of RER corresponds with reverse structural remodeling assessed by echocardiography.
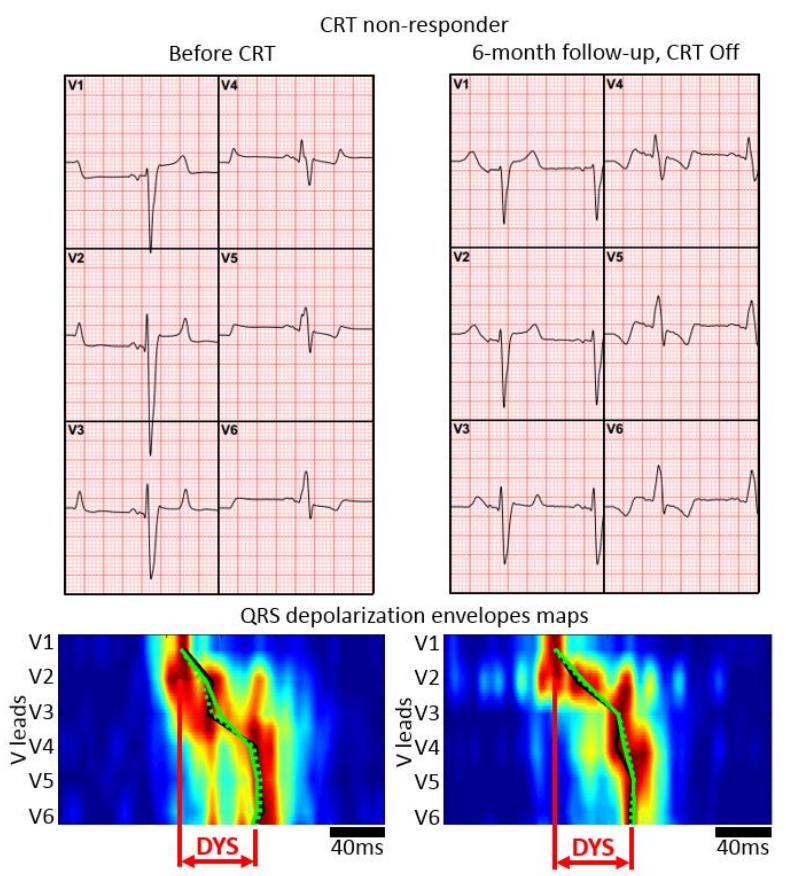

Figure 3. Example of the CRT non-responder by echocardiography (no change of ESV); with almost no change of QRSd (157ms to 160ms) and DYS (61ms to $58 \mathrm{~ms}$ ). QRS depolarization envelopes maps are created by amplitude normalization over the $\mathrm{V}$ lead envelopes (max. amplitude - red, min. amplitude - blue color), arranging envelopes into rows from V1 to V6 and performing a linear interpolation between the rows.

\section{Acknowledgements}

The research was supported by Czech Science Foundation, project GA17-13830S, by Ministry of Education, Youth and Sports of the Czech Republic (project LO1212 and LQ1605) and by the Czech Academy of Sciences (project RVO:68081731).

\section{References}

[1] Azevedo PS, Polegato BF, Minicucci MF, Paiva SA, Zornoff LA. Cardiac remodeling: concepts, clinical impact, pathophysiological mechanisms and pharmacologic treatment. Arq Bras Cardiol 2016;106(1):62-9.

[2] Ghio S, Freemantle N, Scelsi L, Serio A, Magrini G, Pasotti $\mathrm{M}$, et al. Long-term left ventricular reverse remodelling with cardiac resynchronization therapy: results from the
CARE-HF trial. Eur J Heart Fail 2009;11(5):480-8.

[3] Henrikson CA, Spragg DD, Cheng A, Capps M, Devaughn $\mathrm{K}$, Marine JE, et al. Evidence for electrical remodeling of the native conduction system with cardiac resynchronization therapy. Pacing Clin Electrophysiol 2007;30(5):591-5.

[4] Sebag FA, Martins RP, Defaye P, Hidden-Lucet F, Mabo $\mathrm{P}$, Daubert JC, et al. Reverse electrical remodeling by cardiac resynchronization therapy: prevalence and clinical impact. J Cardiovasc Electrophysiol 2012;23(11):1219-27.

[5] Aslani A, Khajei M, Shahrzad S, Nikoo MH, Jorat MV, Bigi MA. Effect of cardiac resynchronisation therapy on electrical remodelling. Heart Lung Circ 2016;25(5):471-5.

[6] Stockburger M, Nitardy A, Fateh-Moghadam S, Krebs A, Celebi O, Karhausen T, et al. Electrical remodeling and cardiac dimensions in patients treated by cardiac resynchronization and heart failure controls. Pacing Clin Electrophysiol 2008;31(1):70-7.

[7] Jurak P, Halamek J, Meluzin J, Plesinger F, Postranecka T, Lipoldova $\mathrm{J}$, et al. Ventricular dyssynchrony assessment using ultra-high frequency ECG technique. J Interv Card Electrophysiol 2017;49(3):245-54.

[8] Vitek M, Hrubes J, Kozumplik J. A Wavelet-Based ECG Delineation with Improved $\mathrm{P}$ Wave Offset Detection Accuracy. Biosig Brno 2010:160-5.

[9] Moss AJ, Hall WJ, Cannom DS, Klein H, Brown MW, Daubert JP, et al. Cardiac-resynchronization therapy for the prevention of heart-failure events. $\mathrm{N}$ Engl $\mathrm{J}$ Med 2009;361(14):1329-38.

[10] Plesinger F, Jurak P, Halamek J, Nejedly P, Leinveber P, Viscor I, et al. Ventricular electrical delay measured from body surface ECGs is associated with cardiac resynchronization therapy response in left bundle branch block patients from the MADIT-CRT trial. Circ Arrhythm Electrophysiol 2018;11(5):e005719.

[11] Waring AA, Litwin SE. Redefining reverse remodeling: can echocardiography refine our ability to assess response to heart failure treatments? J Am Coll Cardiol 2016;68(12):1277-80.

[12] Kamireddy S, Agarwal SK, Adelstein E, Jain S, Saba S. Correlation of electrical and mechanical reverse remodeling after cardiac resynchronization therapy. Ann Noninvasive Electrocardiol 2009;14(2):153-7.

[13] Lellouche N, De Diego C, Boyle NG, Wiener I, Akopyan G, Child JS, et al. Relationship between mechanical and electrical remodelling in patients with cardiac resynchronization implanted defibrillators. Europace 2011;13(8):1180-7.

[14] Molhoek SG, L VANE, Bootsma M, Steendijk P, Van Der Wall EE, Schalij MJ. QRS duration and shortening to predict clinical response to cardiac resynchronization therapy in patients with end-stage heart failure. Pacing Clin Electrophysiol 2004;27(3):308-13.

Address for correspondence.

Pavel Leinveber

St. Anne's University Hospital Brno

International Clinical Research Center

Pekařská 53, 65991 Brno, Czech Republic

pavel.leinveber@fnusa.cz 